\title{
Permutations avoiding an increasing number of length-increasing forbidden subsequences
}

\author{
Elena Barcucci, Alberto Del Lungo, Elisa Pergola, Renzo Pinzani \\ Dipartimento di Sistemi e Informatica, Università di Firenze, Via Lombroso 6/17, 50134 Firenze, Italy, e-mail: bar- \\ cucci,dellungo,elisa,pinzani@dsi.unifi.it
}

\begin{abstract}
A permutation $\pi$ is said to be $\tau$-avoiding if it does not contain any subsequence having all the same pairwise comparisons as $\tau$. This paper concerns the characterization and enumeration of permutations which avoid a set $F^{j}$ of subsequences increasing both in number and in length at the same time. Let $F^{j}$ be the set of subsequences of the form " $\sigma(j+1)(j+2)$ ", $\sigma$ being any permutation on $\{1, \ldots, j\}$. For $j=1$ the only subsequence in $F^{1}$ is 123 and the 123-avoiding permutations are enumerated by the Catalan numbers; for $j=2$ the subsequences in $F^{2}$ are 1234,2134 and the $(1234,2134)$-avoiding permutations are enumerated by the Schröder numbers; for each other value of $j$ greater than 2 the subsequences in $F^{j}$ are $j$ ! and their length is $(j+2)$; the permutations avoiding these $j$ ! subsequences are enumerated by a number sequence $\left\{a_{n}\right\}_{n \geq 1}$ such that $C_{n} \leq a_{n} \leq n$ !, $C_{n}$ being the $n$-th Catalan number. For each $j$ we determine the generating function of permutations avoiding the subsequences in $F^{j}$, according to the length, to the number of left minima and of non-inversions.
\end{abstract}

Keywords: Permutations, Forbidden subsequences, Catalan numbers, Schröder numbers

\section{Introduction}

The study of permutations represents an interesting and relevant discipline in Mathematics which began with Euler who first analyzed permutation statistics related to the study of parameters different from their length [16]. MacMahon in [24] further developed this vast field but meaningful progress has been only made in the last thirty years.

Recently, the new problems coming from Computer Science led to the development of the concept of permutations with forbidden subsequences. They arise in sorting problems [10, 22, 33, 36, 37], in the analysis of regularities in words [4,23], in particular instances of pattern matching algorithms optimization [8]; just to mention some examples. The enumeration of permutations with specific forbidden subsequences has also applications in areas such as Algebraic Geometry and Combinatorics. The 2143avoiding permutations, called vexillary permutations, are relevant to the theory of Schubert polynomials. In Combinatorics, permutations with forbidden subsequences play an important role as they present bijections with a great number of non-trivial combinatorial objects $[13,14,15,18,20,21]$ and moreover their enumeration gives rise to classical number sequences. 
The $n$-th Catalan number is the common value of permutations with a single forbidden subsequence of length three [22]. More precisely Knuth shows that 312-avoiding permutations are the one stack sortable permutations. The problem of avoiding more than one restriction was first studied by Simion and Schmidt [34] and they determined the number of permutations avoiding two or three subsequences of length three.

As far as forbidden subsequences of length four are concerned, new enumeration results concern the subsequence 1234 [17], 1342 [6] and all the ones behaving identically [1, 35, 36], while for 1324-avoiding permutations the only result is proved by Bóna in [5] and it gives only a numerical lower bound. Permutations avoiding some couples of subsequences of length four give the Schröder numbers [20]; results concerning permutations avoiding more than one forbidden subsequence of length four exist; we refer to [20] for an exhaustive survey of the results available on permutations with forbidden subsequences.

Regarding permutations avoiding a single subsequence of length greater than four the most important result solves the problem of one increasing subsequence of any length giving an asymptotic value of the number of permutations avoiding the subsequence $1 \ldots k+1$ [30]. In [11] Chow and West study $(123, k \ldots 1(k+1))$-avoiding permutations; their generating functions can be expressed as a quotient of modified Chebyshev polynomials and they give rise to number sequences lying between the well-known Fibonacci and Catalan numbers. In [3] the authors study $(321,(k+2) \overline{1}(k+3) 2 \ldots(k+1))$-avoiding permutations where the latter forbidden subsequence means that the subsequence $(k+1)(k+2) 1 \ldots k$ [that is $(k+2)(k+3) 2 \ldots k+1$ restricted on $\{1, \ldots, k+2\}$ ] is allowed only in the case it is part of a longer subsequence of type $(k+2) 1(k+3) 2 \ldots k+1$. Their generating functions are algebraic and give rise to sequences of numbers lying between the well-known Motzkin and Catalan numbers involving the left-Motzkin factor numbers as a particular case.

A natural generalization of sequence avoidance is the restricted sequence inclusion. In this case a prescribed number of occurences of a sequence in the permutations is required. Noonan [28] and Bona [7] determined a simple expression for the number of permutations containing exactly one 123 , respectively one 132 sequence. Robertson [31] proved that the number of 123-avoiding permutations containing exactly one 132 sequence is given by $(n-2) 2^{n-3}$. There are some other recent interesting results. Robertson, Wilf and Zeilberger [32] express the generating function for the number of 132-avoiding permutations which have a given number of 123 sequences in form of a continued fraction. Mansour and Vainshtein [27] extend the previous result to determine the generating function of the number of 132-avoiding permutations having a given number of $12 \ldots k$ sequences. Mansour [25] studies the permutations avoiding a sequence of length four and a nonempty set of sequences of length three. Mansour [26] provides a simple espression for the number of permutations which avoid all the sequences of $\left\{\tau \in S_{k} \mid \tau_{1}=m\right\}$ (i.e., the sequences of length $k$ having the first element equal to $m$ ). Moreover, he gives a generalization of Robertson's result.

In this paper, we continue this line of research in counting permutations which avoid a set of restrictions. We study a case which is similar to the problem studied by Mansour [26], that is the set of permutations which avoid all the sequences of $\left\{\tau \in S_{j+2} \mid \tau_{j+1}=j+1, \tau_{j+2}=j+2\right\}$.

Section 2 of this paper contains the basic definitions about permutations with forbidden subsequences. In Section 3, we describe the tools used to obtain the enumerative results, which are succession rules and generating trees. The former ones consist of rules describing the growing behavior of an object with a fixed parameter value, the latter ones are schematic representations of the former. In Section 4, we express the permutations we are studying in terms of succession rules. We translate the construction, represented by the generating tree, into formulae, thus obtaining a set of functional equations. Their solution gives the generating function of the permutations according to the length, number of left minima, 
non-inversions and active sites. We are able to determine the generating function according to the length of the permutations, number of left minima and non-inversions. This result allows us to show that the generating function is algebraic of degree two, except for $j=\infty$.

\section{Notations and definitions}

In this section we recall the basic definitions about permutations with forbidden subsequences that will be referred to in the next sections.

A permutation $\pi=\pi(1) \pi(2) \ldots \pi(n)$ on $[n]=\{1,2, \ldots, n\}$ is a bijection between $[n]$ and $[n]$. Let $S_{n}$ be the set of permutations on $[n]$.

A permutation $\pi \in S_{n}$ contains a subsequence of type $\tau \in S_{k}$ if and only if a sequence of indices $1 \leq i_{\tau(1)}<i_{\tau(2)}<\ldots<i_{\tau(k)} \leq n$ exists such that $\pi\left(i_{1}\right) \pi\left(i_{2}\right) \ldots \pi\left(i_{k}\right)$ has all the same pairwise comparisons as $\tau$. We denote the set of permutations of $S_{n}$ not containing subsequences of type $\tau$ by $S_{n}(\tau)$.

Example 2.1 The permutation 6145732 belongs to $S_{7}(2413)$ because all its subsequences of length 4 are not of type 2413 . This permutation does not belong to $S_{7}(3142)$ because there exist subsequences of type 3142 , namely $\pi(1) \pi(2) \pi(5) \pi(6)=6173, \pi(1) \pi(2) \pi(5) \pi(7)=6172$.

If we have the set $\tau_{1} \in S_{k_{1}}, \ldots, \tau_{p} \in S_{k_{p}}$ of permutations, we denote the set $S_{n}\left(\tau_{1}\right) \cap \ldots \cap S_{n}\left(\tau_{p}\right)$ by $S_{n}\left(\tau_{1}, \ldots, \tau_{p}\right)$. We call the family $F=\left\{\tau_{1}, \ldots, \tau_{p}\right\}$ a family of forbidden subsequences, the set $S_{n}(F)$ a family of permutations with forbidden subsequences and $\bigcup_{n \geq 1} S_{n}(F)$ a class of permutations with forbidden subsequences.

Given $\pi \in S_{n}$, we call the position on the left of $\pi(1)$ position 0 , the position between $\pi(i)$ and $\pi(i+1)$, $1 \leq i \leq n-1$, position $i$, and the position on the right of $\pi(n)$, position $n$. We will refer to any of these positions as the sites of $\pi$.

Definition 2.1 Let $F=\left\{\tau_{1}, \ldots, \tau_{p}\right\}$. The position $i, 0 \leq i \leq n$, of a permutation $\pi \in S_{n}(F)$ is an active site if the insertion of $n+1$ into position $i$ gives a permutation belonging to the set $S_{n+1}(F)$; otherwise it is said to be an inactive site.

Example 2.2 The permutation $\pi=6145732 \in S_{7}(2413)$ has 5 active sites (the positions 0, 4, 5, 6 and 7 ) and 3 inactive sites (the positions 1,2 and 3 ) as $61458732 \in S_{8}(2413)$ while $61845732 \notin S_{8}(2413)$, just to give an example.

Definition 2.2 Let $\pi \in S_{n}$. The pair of indices $(i, j), i<j$, is a non-inversion if $\pi(i)<\pi(j)$. An element $\pi(i)$ is a left minimum if $\pi(i)<\pi(j), \forall j \in[1, i-1]$.

Example 2.3 The permutation of Example 2.2 has 9 non-inversions: $(1,5)(2,3)(2,4)(2,5)(2,6)$ $(2,7)(3,4)(3,5)(4,5)$ and 2 left minima: $\pi(1)=6$ and $\pi(2)=1$.

\section{Succession rules and generating trees}

In this section we briefly describe the tools used to deduce our enumerative results, namely succession rules and generating trees. They were introduced in [12] for the study of Baxter permutations and further applied to the study of permutations with forbidden subsequences by West [11, 38, 39]. 
Definition 3.1 A generating tree is a rooted, labelled tree such that the labels of the set of children of each node $\mathrm{v}$ can be determined from the label of $\mathrm{v}$ itself. Thus, any particular generating tree can be specified by a recursive definition consisting of:

1. basis: the label of the root,

2. inductive step: a set of succession rules that yields a multiset of labelled children depending solely on the label of the parent.

A succession rule contains at least the information about the number of children. Let $\tau$ be a forbidden subsequence. Following the idea developed in [12], the generating tree for $\tau$-avoiding permutations is a rooted tree such that the nodes on level $n$ are exactly the elements of $S_{n}(\tau)$; the children of a permutation $\pi=\pi(1) \ldots \pi(n)$ are all the $\tau$-avoiding permutations obtained by inserting $n+1$ into $\pi$. Labels must be assigned to the nodes and they record the number of children of a given node.

Example 3.1 The Catalan tree and 123-avoiding permutations are obtained by the succession rule:

$$
\left\{\begin{array}{l}
\text { basis: } \\
\text { inductive step : }
\end{array}(k) \rightarrow(k+1)(2) \ldots(k) .\right.
$$

The permutation of length one has two active sites (basis in rule (3.1)). Let $\pi=\pi(1) \ldots \pi(n) \in S_{n}(123)$; and let $k, 2 \leq k \leq n$, be the minimum index in $\pi$ such that $i_{1}<k$ exists and $\pi\left(i_{1}\right)<\pi(k)$; then the active sites of $\pi$ are the positions $0, \ldots, k-1$. The insertion of $n+1$ into each other site on the right of the position $k-1$ gives the subsequence $\pi\left(i_{1}\right) \pi(k)(n+1)$ that is forbidden. This means that the active sites of $\pi$ are all the positions lying among any pair of elements of $\pi$ constituting the longest initial decreasing subsequence. If $\pi$ has $k$ active sites then its longest initial decreasing subsequence has length $k-1$. The permutation obtained by inserting $n+1$ into the position 0 gives a new permutation with $(k+1)$ active sites; the permutation obtained by inserting $n+1$ into the position $i, 1 \leq i \leq k-1$, gives $(i+1)$ active sites, (inductive step in rule (3.1)). The generating tree representing 123-avoiding permutations can be obtained by developing rule (3.1) and by labelling each permutation with the right label $(k)$.

Example 3.2 The Schröder tree and $(1234,2134)$-avoiding permutations are obtained by the succession rule:

$$
\begin{cases}\text { basis : } & (2) \\ \text { inductive step }: & (2) \rightarrow(3)(3) \\ \text { inductive step }: & (k) \rightarrow(k+1)(k+1)(3) \ldots(k), \quad k \geq 3 .\end{cases}
$$

The permutation of length one has two active sites (basis in rule (3.2)). Let $\pi=\pi(1) \ldots \pi(n) \in S_{n}(1234$, 2134); and let $k, 3 \leq k \leq n$, be the minimum index in $\pi$ such that there exist $i_{1}<i_{2}<k$ for which $\pi\left(i_{1}\right) \pi\left(i_{2}\right) \pi(k)$ is of type 123 , or 213 ; then the active sites of $\pi$ are the positions $0, \ldots, k-1$. The insertion of $n+1$ into each other site, that is the positions $k, \ldots, n$, gives at least one of the forbidden subsequences 1234,2134 . Let $\pi$ be a permutation with $k$ active sites; the permutations obtained by inserting $n+1$ into the position 0 and 1 have $(k+1)$ active sites; the permutation obtained by inserting $n+1$ into the position $i$, $2 \leq i \leq k-1$, has $(i+1)$ active sites, each other site gives at least one of the two forbidden subsequences because $n+1$ has at least two smaller elements on its left (inductive step in rule (3.2)). The generating tree related to rule (3.2) and representing $(1234,2134)$-avoiding permutations is shown in Figure 1. 


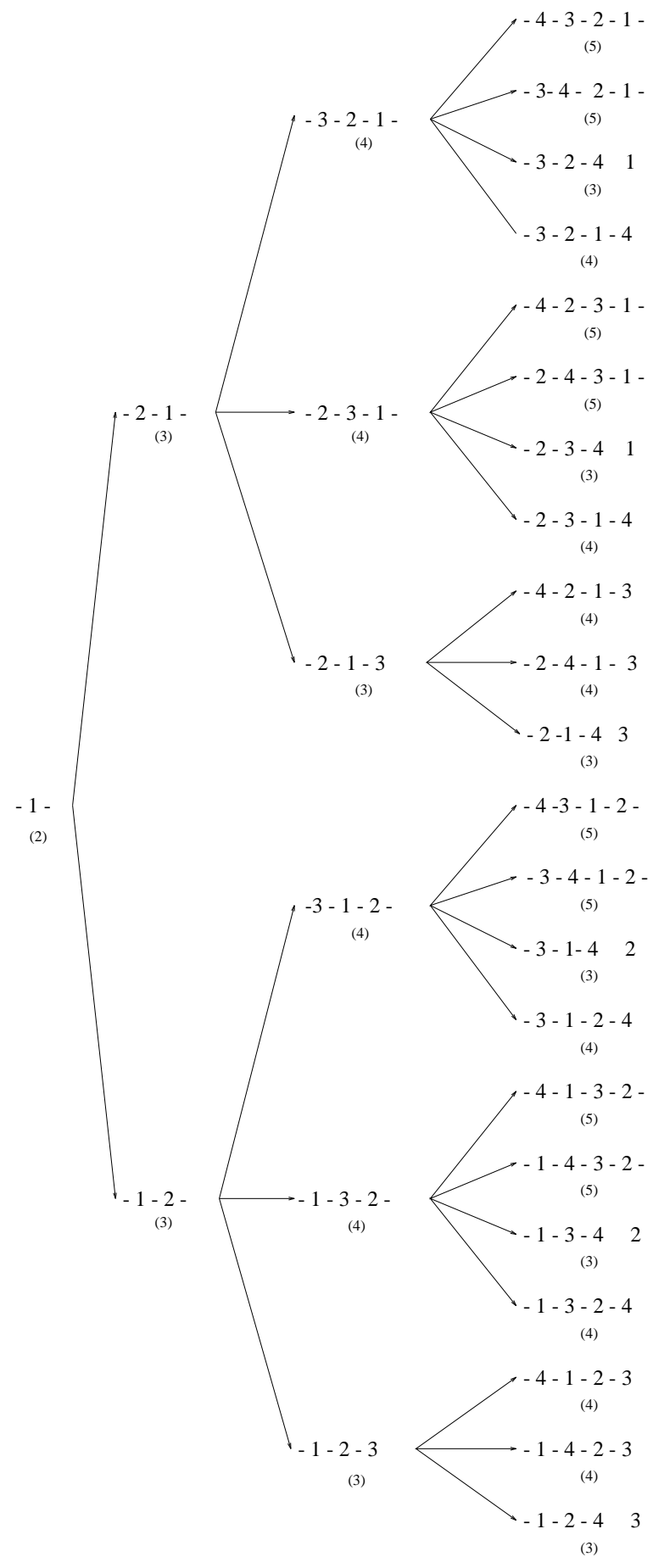

Fig. 1: The generating tree for $(1234,2134)$-avoiding permutations. 
It should be noticed that the succession rule (3.2) differs from (3.1) just by a replacement of (2) by $(k+1)$ in the inductive step.

\section{Permutations avoiding $S_{j}(j+1)(j+2)$}

In this section we study the class of permutations $\mathcal{C}^{j}=\bigcup_{n \geq 1} S_{n}\left(F^{j}\right)$, where $F^{j}$ is a set of subsequences such that $\left|F^{j}\right|=j$ ! and any $\tau \in F^{j}$ has the form $\tau=\sigma(j+1)(j+2), \sigma$ being a permutation belonging to the symmetric group $S_{j}$. Another way to characterize the class of permutations $\mathcal{C}^{j}$ is in terms of patterns in the corresponding permutation matrix. In this view what is forbidden is any submatrix of the form:

$$
\left(\begin{array}{ccccc}
1 & 0 & \cdots & 0 & 0 \\
0 & 0 & \cdots & 0 & 0 \\
\vdots & & & & \vdots \\
0 & 0 & \cdots & 0 & 1
\end{array}\right)
$$

with as many as $j 1$ 's appearing in both above and to the left of this structure.

These permutations are enumerated by number sequences such that the $n$-th term is between the $n$-th Catalan number and $n$ ! (see Figure 2). We describe the structure of their generating tree and use this construction to obtain a set of functional equations satisfied by the generating function of the class of permutations $\mathcal{C}^{j}$. Some computations allow us to determine this generating function according to the length of the permutations, number of left minima and non-inversions.

\section{From Catalan numbers to factorial}

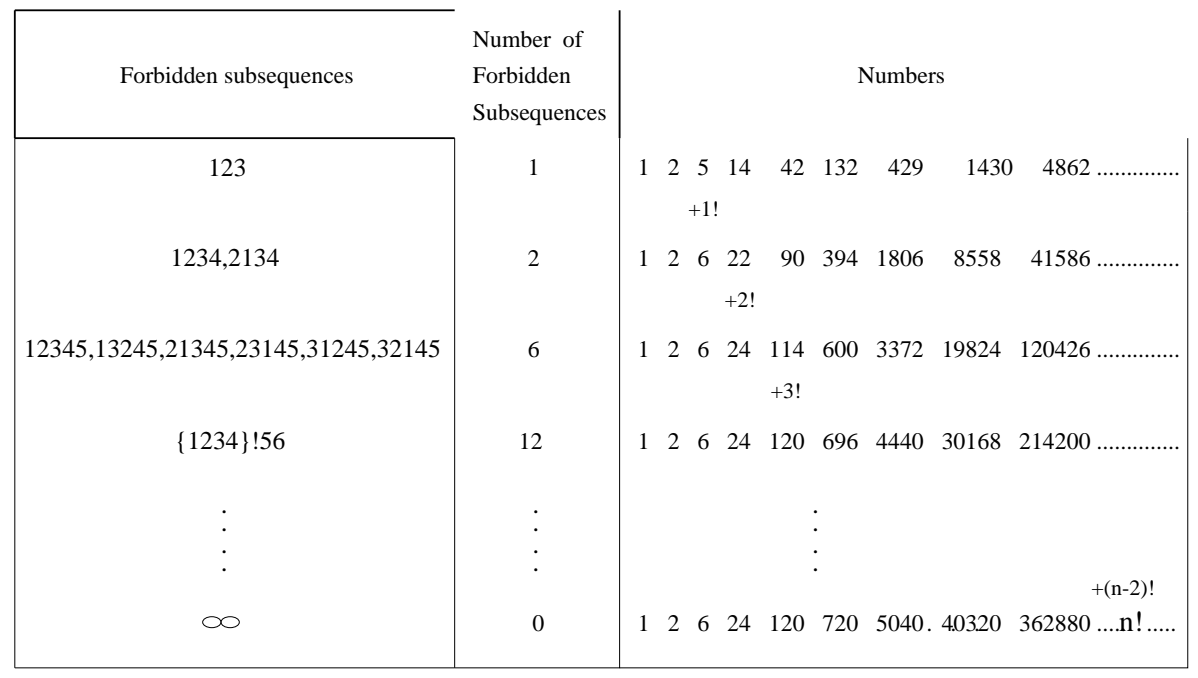

Fig. 2: First numbers of the sequences counting the permutations in $\mathcal{C}^{j}$. 


\subsection{The generating tree for $\mathcal{C}^{j}$ permutations}

The class of permutations $\mathcal{C}^{j}$ contains permutations avoiding configurations of the form $\ldots x \ldots y \ldots$ such that $x<y$ and $x$ is preceded by at least $j$ elements all of them less than $x$. As a matter of fact, the set $F^{j}$ contains subsequences having length $(j+2)$ such that the two largest elements, that is $(j+1),(j+2)$, are the $(j+1)$-th and the $(j+2)$-th elements of the sequences, while the other $j$ elements $\{1, \ldots, j\}$ can be in any order. This means that the position $i, 0 \leq i \leq n$, in a permutation $\pi \in S_{n}\left(F^{j}\right)$, is an active site if and only if a sequence of indices $i_{1}, \ldots, i_{j}, i_{j+1}$ such that $i_{1}<\cdots<i_{j}<i_{j+1} \leq i$ and $\max _{1 \leq t \leq j}\left(\pi\left(i_{t}\right)\right)<\pi\left(i_{j+1}\right)$ does not exist. It follows that the active sites lie on the left of the minimum index $\bar{i}_{j+1}$ that gives such a sequence. This, in turn, means that the $(j+1)$ leftmost sites of a permutation are always active, if they exist.

Let $\pi \in S_{n}\left(F^{j}\right), j \geq 1$, be a permutation with $k$ active sites, the positions $0, \ldots, k-1$, so there is a sequence of indices $i_{1}, \ldots, i_{j+1}=k$ such that $i_{1}<\cdots<i_{j}<k$ and $\max _{1 \leq t \leq j} \pi\left(i_{t}\right)<\pi(k)$. By inserting $n+1$ into the position $i, 0 \leq i \leq j-1$, we obtain a new permutation, $\pi^{i}$, with $(k+1)$ active sites as the new element is not relevant to the existence of the sequence of indices $i_{1}, \ldots, i_{j}, i_{j+1}$, and the minimum index that gives such a sequence is $(k+1)$ as the $(k+1)$-th element of $\pi^{i}$ is equal to $\pi(k)$. By inserting $n+1$ into the position $i, j \leq i \leq k-1$, we obtain a new permutation $\pi^{i}$ with $(i+1)$ active sites. As a matter of fact, the sequence of indices $1, \ldots, j,(i+1)$ satisfies the required properties and moreover $(i+1)$ is the minimum index leading to such a sequence (see Figure 3 ).

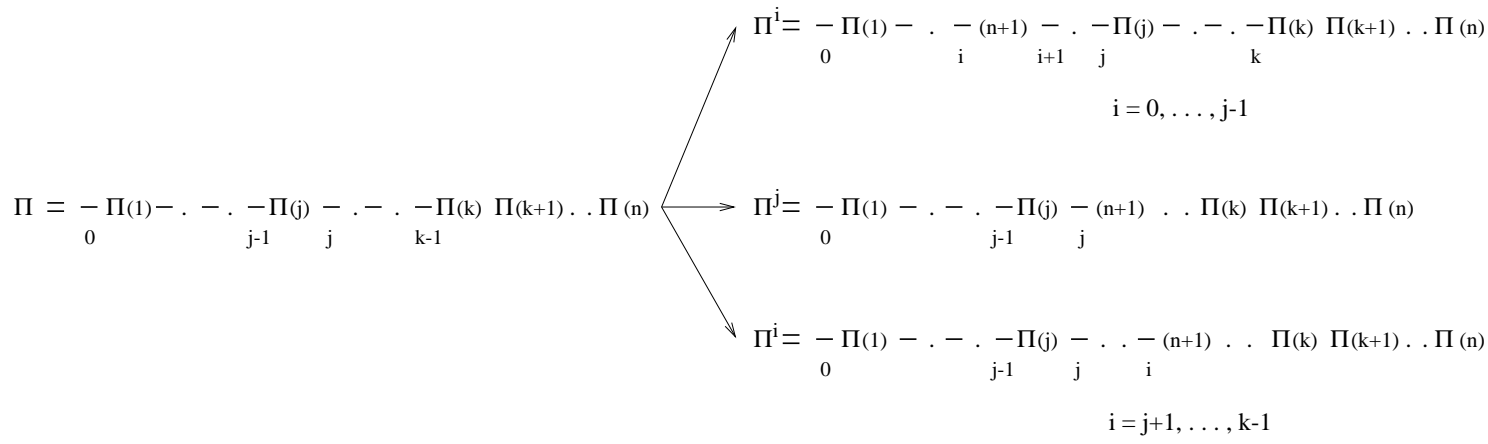

Fig. 3: The active sites in the children of $\pi$.

The above arguments prove the following proposition:

Proposition 4.1 Let $\pi \in S_{n}\left(F^{j}\right), j \geq 1$, be a permutation with $k$ active sites (namely the positions $0, \ldots, k-1)$, and let $\pi^{i}$ be the permutation obtained from $\pi$ by inserting $n+1$ into $i$, then the number of active sites of $\pi^{i}$ is $(k+1)$ for $0 \leq i \leq j-1$ and $(i+1)$ for $j \leq i \leq k-1$.

The succession rule for the generating tree of $\mathcal{C}^{j}$ permutations follows immediately:

$$
\left\{\begin{array}{lll}
\text { basis : } & \\
\text { inductive step }: & (k) \rightarrow(k+1)^{k}, & k<j \\
\text { inductive step }: & (k) \rightarrow(k+1)^{j}(j+1) \ldots .(k), & k \geq j .
\end{array}\right.
$$


In a "Catalan permutation" $\pi$ a position $i, 0 \leq i \leq n$, is an active site if and only if there do not exist any $i_{i}<i_{2} \leq i$ such that $\pi\left(i_{1}\right)<\pi\left(i_{2}\right)$; in a "Schröder permutation" $\pi$ a position $i, 0 \leq i \leq n$, is an active site if and only if there do not exist any $i_{1}<i_{2}<i_{3} \leq i$ such that $\max \left\{\pi\left(i_{1}\right), \pi\left(i_{2}\right)\right\}<\pi\left(i_{3}\right)$. By comparing these conditions with the definition of active site for a permutations $\pi \in \mathcal{C}^{j}$; we realise that the role of the parameter $j$ is to increase the length of the index sequence $i_{1}, \ldots, i_{j+1}$ such that $i_{1}<\ldots<i_{j}<i_{j+1}$ and each $\pi\left(i_{t}\right), t=1, \ldots, j$, is smaller than $\pi\left(i_{j+1}\right)$. Observe that no constraint is required on the order of the elements of the set $\left\{\pi\left(i_{1}\right), \ldots, \pi\left(i_{j}\right)\right\}$. Once the permutations in $\mathcal{C}^{j}$ are established we are interested in the results concerning their enumeration.

\subsection{The generating function}

For each $j$, we are interested in the generating function for the permutations in $\mathcal{C}^{j}$ according to the length, number of left minima and non-inversions. Let $\pi \in \mathcal{C}^{j}$; we denote the length of $\pi$ by $n(\pi)$, the number of its left minima by $m(\pi)$, the number of its non-inversions by $n i(\pi)$ and the number of its active sites by $a(\pi)$. The generating function of $\mathcal{C}^{j}$ according to the above mentioned parameters is the following:

$$
C^{j}(x, y, q, s)=\sum_{\pi \in \mathcal{C}^{j}} x^{n(\pi)} y^{m(\pi)} q^{n i(\pi)} s^{a(\pi)} .
$$

From (4.3) we deduce that a permutation $\pi \in \mathcal{C}^{j}$ is the father of $a(\pi)$ permutations, namely $\pi^{0}, \ldots$, $\pi^{a(\pi)-1}$ in $\mathcal{C}^{j}$, obtained by inserting a new element into the positions $0, \ldots, a(\pi)-1$ which are active sites. If we look at the parameter changes in each permutation $\pi^{i}, 0 \leq i \leq a(\pi)-1$, then we find:

a) if $0 \leq i \leq \min \{j, a(\pi)\}-1$ then:

$$
n\left(\pi^{i}\right)=n(\pi)+1, \quad\left\{\begin{array}{l}
m\left(\pi^{0}\right)=m(\pi)+1, \\
m\left(\pi^{i}\right)=m(\pi) \text { if } i>0, \quad n i\left(\pi^{i}\right)=n i(\pi)+i, \quad a\left(\pi^{i}\right)=a(\pi)+1 .
\end{array}\right.
$$

b) if $\min \{j, a(\pi)\} \leq i \leq a(\pi)-1$ then:

$$
n\left(\pi^{i}\right)=n(\pi)+1, \quad m\left(\pi^{i}\right)=m(\pi), \quad n i\left(\pi^{i}\right)=n i(\pi)+i, \quad a\left(\pi^{i}\right)=i+1 .
$$

The set $\mathcal{C}^{j}$ can be partitioned into $j$ subsets: $\mathcal{C}^{(j, 2)}, \ldots, \mathcal{C}^{(j, j)}, \mathcal{C}^{(j,>)}$, where $\mathcal{C}^{(j, k)}=\left\{\pi \in \mathcal{C}^{j}\right.$ : $a(\pi)=k\}, 2 \leq k \leq j$, and $\mathcal{C}^{(j,>)}=\left\{\pi \in \mathcal{C}^{j}: a(\pi)>j\right\}$. In terms of generating functions we obtain the decomposition:

$$
C^{j}(x, y, q, s)=\sum_{k=2}^{j} C^{(j, k)}(x, y, q, 1) s^{k}+C^{(j,>)}(x, y, q, s) .
$$

Let $\mathcal{C}^{(j, 1)}$ be the set representing the permutation of length 0 . Observe that each permutation $\pi$ such that $a(\pi) \leq j-1$ gives $a(\pi)$ permutations with $(a(\pi)+1)$ active sites; so $\mathcal{C}^{(j, k)}$ is given by modifying $\mathcal{C}^{(j, k-1)}, 2 \leq k \leq j$, and the parameter changes are described in a). This means that a permutation of $\mathcal{C}^{j}$ having exactly $k \leq j$ active sites is just any permutation of length $k-1$. Therefore each permutation $\pi$ such that $a(\pi) \geq j$ gives $a(\pi)$ permutations with at least $(j+1)$ active sites and the parameter changes are described in a) for the $j$ leftmost active sites and in $\mathbf{b}$ ) for the remaining ones.

By translating the above arguments into formulae we obtain: 


$$
\begin{aligned}
C^{(j, 1)}(x, y, q, s) & =s \\
C^{(j, k)}(x, y, q, s) & =\left(x y s+\frac{x q s}{q-1}\left(q^{k-2}-1\right)\right) C^{(j, k-1)}(x, y, q, s), 2 \leq k \leq j \\
C^{(j,>)}(x, y, q, s) & =\left(x y s+\frac{x q s}{q-1}\left(q^{j-1}-1\right)\right) C^{(j, j)}(x, y, q, s)+ \\
& +\left(x y s+\frac{x q s}{q-1}\left(q^{j-1}-1\right)\right) C^{(j,>)}(x, y, q, s)+ \\
& +\frac{x s^{j+1} q^{j}}{1-s q} C^{(j,>)}(x, y, q, 1)-\frac{x s}{1-s q} C^{(j,>)}(x, y, q, s q) .
\end{aligned}
$$

Using the standard notation for the $q$-analogue of the number $i:[i]_{q}=\frac{1-q^{i}}{1-q}$, we obtain the following proposition.

Proposition 4.2 The generating function $C^{j}(x, y, q, s)$ of permutations in $\mathcal{C}^{j}$ is:

$$
C^{j}(x, y, q, s)=\sum_{i=2}^{j} C^{(j, i)}(x, y, q, s)+C^{(j,>)}(x, y, q, s)
$$

where:

$$
\begin{aligned}
C^{(j, 1)}(x, y, q, s) & =s \\
C^{(j, k)}(x, y, q, s) & =x^{k-1} s^{k} y \prod_{i=1}^{k-2}\left(y+q[i]_{q}\right), 2 \leq k \leq j \\
C^{(j,>)}(x, y, q, s) & =\frac{x s\left(y+q[j-1]_{q}\right)}{1-x s\left(y+q[j-1]_{q}\right)} C^{(j, j)}(x, y, q, s)+ \\
& +\frac{x s}{(1-s q)\left(1-x s\left(y+q[j-1]_{q}\right)\right)}\left[s^{j} q^{j} C^{(j,>)}(x, y, q, 1)-C^{(j,>)}(x, y, q, s q)\right] .
\end{aligned}
$$

The third equation of Proposition 4.2 can be solved by using the lemma of Bousquet-Mélou [9]. Let us denote the generating function $F(x, y, q, 1)$ by $F(x, y, q)$.

Proposition 4.3 The generating function $C^{(j,>)}(x, y, q, s)$ is given by:

$$
C^{(j,>)}(x, y, q, s)=\frac{J_{1, j}(x, y, q)}{J_{0, j}(x, y, q)}
$$

where:

$$
\begin{gathered}
J_{1, j}(x, y, q)=\sum_{n \geq 0} \frac{(-1)^{n} x^{n+1} q^{j n+\left(\begin{array}{c}
n+1 \\
2
\end{array}\right)}}{(q)_{n}\left(x\left(y+q[j-1]_{q}\right)\right)_{n+1}}\left(y+q[j-1]_{q}\right) C^{(j, j)}(x, y, q) \\
J_{0, j}(x, y, q)=1+\sum_{n \geq 0} \frac{(-1)^{n+1} x^{n+1} q^{j(n+1)+\left(\begin{array}{c}
n+1 \\
2
\end{array}\right)}}{(q)_{n+1}\left(x\left(y+q[j-1]_{q}\right)\right)_{n+1}}
\end{gathered}
$$

with $(a)_{n}=\prod_{k=0}^{n-1}\left(1-a q^{k}\right)$.

From Proposition 4.3 it follows: 
Theorem 4.4 The generating function $C^{j}(x, y, q)$ of permutations in $\mathcal{C}^{j}$ according to the length, number of left minima and non-inversions is:

$$
C^{j}(x, y, q)=x^{j} y f_{j}(x, y, q) \prod_{i=1}^{j-1}\left(y+q[i]_{q}\right)+\sum_{k=2}^{j} x^{k-1} y \prod_{i=1}^{k-2}\left(y+q[i]_{q}\right), \quad j \geq 1 ;
$$

with:

$$
f_{j}(x, y, q)=\frac{\sum_{n \geq 0} \frac{(-1)^{n} x^{n} q^{j n+\left(\begin{array}{c}
n+1 \\
2
\end{array}\right)}}{(q)_{n}\left(x\left(y+q[j-1]_{q}\right)\right)_{n+1}}}{\sum_{n \geq 0} \frac{(-1)^{n} x^{n} q^{j n+(}\left(\begin{array}{l}
n \\
2
\end{array}\right)}{(q)_{n}\left(x\left(y+q[j-1]_{q}\right)\right)_{n}}} .
$$

We denote the numerator and the denominator of $f_{j}(x, y, q)$ by $A_{j}(x, y, q)$ and $B_{j}(x, y, q)$, respectively. After some computations we obtain:

Lemma 4.5 The functions $A_{j}(x, y, q)$ and $B_{j}(x, y, q)$ satisfy the following equalities:

$$
\begin{gathered}
A_{j}(x q, y, q)=\frac{1-x\left(y+q[j-1]_{q}\right)}{x^{2} q^{j+1}\left(y+q[j-1]_{q}\right)}\left[\left(1-x\left(y+q[j-1]_{q}+q^{j}\right)\right) A_{j}(x, y, q)-B_{j}(x, y, q)\right], \\
B_{j}(x q, y, q)=\left(1-x\left(y+q[j-1]_{q}\right)\right) A_{j}(x, y, q) .
\end{gathered}
$$

Lemma 4.5 allows us to find the $q$-equation satisfied by $f_{j}(x, y, q)$ :

$x^{2} q^{j+1}\left(y+q[j-1]_{q}\right) f_{j}(x q, y, q) f_{j}(x, y, q)-\left(1-x\left(y+q[j-1]_{q}+q^{j}\right)\right) f_{j}(x, y, q)+1=0$.

If $y=q=1$ then Equation (4.4) gives:

$$
f_{j}(x, 1,1)=\frac{1-(j+1) x-\sqrt{1-2(j+1) x+(j-1)^{2} x^{2}}}{2 j x^{2}}
$$

so from Theorem 4.4 we obtain:

$$
C^{j}(x, 1,1)=x^{j-2}(j-1) ! \frac{1-(j+1) x-\sqrt{1-2(j+1) x+(j-1)^{2} x^{2}}}{2}+\sum_{k=1}^{j-1} k ! x^{k} .
$$

This means that the generating function of $\mathcal{C}^{j}$ permutations according to the length is algebraic and quadratic, except for $j=\infty$. In this case we obtain the expected result for the generating function of permutations according to the length, number of left minima and non-inversions, that is:

$$
C^{\infty}(x, y, q)=\sum_{n \geq 1} x^{n} y \prod_{m=1}^{n-1}\left(y+q[m]_{q}\right)
$$




\section{Related results}

The classes of permutations described in this paper are enumerated by numbers lying between the Catalan numbers and the factorial (see Figure 2). The present result is an extension of previously known results for $j=1$ and $j=2$. It is easy to prove that the $(j+2)$-th number of the $(j+1)-$ th sequence is obtained from the $(j+2)$-th term of the $j$-th sequence by adding $j$ !.

In [11], permutations with the forbidden subsequnces $(123,(j+1) j \ldots 1(j+2)), j \geq 1$, are studied. Their generating tree can be expressed in terms of the following succession rule:

$$
\begin{cases}\text { basis : } & (2) \\ \text { inductive step }: & (k) \rightarrow(2) \ldots(k)(k+1), k<j+1 \\ \text { inductive step }: & (j) \rightarrow(2) \ldots(j)(j), k \geq j+1 .\end{cases}
$$

The parameter $j$ allows us to obtain classes of permutations such that the number of permutations of length $n$ is a number lying between $2^{n-1}$ and the $n$-th Catalan number, $C_{n}$. The generating functions of these number sequences are all rational, except for $j=\infty$ as 123 -avoiding permutations are enumerated by the Catalan numbers whose generating function is algebraic.

In [3], permutations with the forbidden subsequnces $(321,(j+1) \overline{1}(j+3) 2 \ldots(j+1)), j \geq 1$, are studied. Their generating tree can be expressed in terms of the following succession rule:

$$
\begin{cases}\text { basis : } & (2) \\ \text { inductive step }: & (k) \rightarrow(k) \ldots(2)(k+1), k<j+1 \\ \text { inductive step }: & (k) \rightarrow(k-1) \ldots(j)(j) \ldots(2)(k+1), k \geq j+1 .\end{cases}
$$

The parameter $j$ allows us to obtain classes of permutations enumerated by numbers lying between the Motzkin and the Catalan numbers whose generating functions are both algebraic.

In [29], the set of permutations $S\left(\bar{F}_{1}^{j}\right) \cup \cdots \cup S\left(\bar{F}_{j}^{j}\right)$, where $\bar{F}_{i}^{j}=(j+3) \bar{i}(j+2) \sigma_{i}$ and $\sigma_{i}$ is a permutation on the set $\{(j+1), \ldots,(i+1),(i-1), \ldots, 1\}$ is introduced. The resulting generating tree is described by the succession rule:

$$
\begin{cases}\text { basis : } & (2) \\ \text { inductive step }: & (k) \rightarrow(k) \ldots(k+1)^{k}, k<j+1 \\ \text { inductive step }: & (k) \rightarrow(k-1)^{k-j}(k+1)^{j}, \quad k \geq j+1 .\end{cases}
$$

The parameter $j$ allows us to obtain classes of permutations enumerated by numbers lying between the Bell numbers and the factorial both having transcendent generating functions.

Let us note that we describe countably many succession rules which lead to rational, algebraic and transcendent generating functions. These are instances of the general theory developed by $\mathrm{C}$. Banderier et al. in [2]. 


\section{References}

[1] E. Babson, J. West, The permutations $123 p_{4} \ldots p_{l}$ and $321 p_{l} \ldots p_{1}$ are Wilf equivalent, Graph and Combinatorics (to appear).

[2] C. Banderier, M. Bousquet-Mélou, A. Denise, P. Flajolet, D. Gardy, D. Gouyou-Beauchamps, On generating functions of generating trees, Proceedings of $11^{\text {th }}$ FPSAC (Barcelona) (1999).

[3] E. Barcucci, A. Del Lungo, E. Pergola, R. Pinzani, From Motzkin to Catalan permutations, Discrete Mathematics (to appear).

[4] J. Berstel, Axel Thue's papers on repetitions in words: translation, Publication du LaCIM 19 (1994).

[5] M. Bóna, Permutations avoiding certain patterns. The case of length 4 and some generalizations, Discrete Mathematics, 175 (1997) 55-67.

[6] M. Bóna, Exact enumeration of 1342-avoiding permutations; a close link with labelled trees and planar maps, Journal of Combinatorial Theory, Ser A, 80 (1997) 257-272.

[7] M. Bona, Permutations with one or two 132-sequences, Discrete Mathematics, 181 (1998) 267-274.

[8] P. Bose, J.F. Buss, A. Lubiw, Pattern matching for permutations, Lecture Notes in Computer Science, 709 (1993) 200-209.

[9] M. Bousquet-Mélou, A method for the enumeration of various classes of column-convex polygons, Discrete Mathematics, 154 (1996) 1-25.

[10] M. Bousquet-Mélou, Multi-statistic enumeration of two-stack sortable permutations, Electronic Journal of Combinatorics, 5 (1998) \#R21.

[11] T. Chow, J. West, Forbidden subsequences and Chebyshev polynomials, Discrete Mathematics, 204 (1999) 119-128.

[12] F.R.K. Chung, R.L. Graham, V.E. Hoggatt, M. Kleiman, The number of Baxter permutations, Journal of Combinatorial Theory, Ser. A, 24 (1978) 382-394.

[13] R. Cori, S. Dulucq, G. Viennot, Shuffle of parenthesis systems and Baxter permutations, Journal of Combinatorial Theory, Ser. A, 43 (1986) 1-22.

[14] S. Dulucq, S. Gire, J. West, Permutations à motifs exclus et cartes planaires non séparables, Proceedings of $5^{\text {th }}$ FPSAC, Florence (1993) 165-178.

[15] S. Dulucq, O. Guibert, Mots de piles, tableaux standards et permutations de Baxter, Proceedings of $6^{\text {th }}$ FPSAC, Dimacs (1994) 119-128.

[16] L. Euler, Institutiones calculi differentialis, in Opera Omnia, Series Prima, vol. X, Verlag von B.G. Teubner, Leipzig (1913).

[17] I.M. Gessel, Symmetric functions and P-recursiveness, Journal of Combinatorial Theory, Ser. A, 53 (1990) 257-285. 
[18] S. Gire, Arbres, permutations a motifs exclus et cartes planaires: quelques problemes algorithmiques et combinatoires, Thèse de l'Université de Bordeaux I (1993).

[19] R.L. Graham, D.E. Knuth, O. Patashnik, Concrete Mathematics, Addison-Wesley Pub. Co., Reading, Massachusetts (1989).

[20] O. Guibert, Combinatoires des permutations a motifs exclus en liaison avec mots, cartes planaires et tableaux de Young, Thèse de l'Université de Bordeaux I (1996).

[21] I. P. Goulden, J. West, Raney paths and a combinatorial relationship between rooted nonseparable planar maps and two-stack-sortable permutations, Journal of Combinatorial Theory, Ser. A (to appear).

[22] D.E. Knuth, The art of computer programming, vol. 3, Addison-Wesley, Reading, MA (1973).

[23] M. Lothaire, Combinatorics and words, G.C. Rota éditor, Encyclopedia of Mathematics and its Applications 17, Addison-Wesley, Reading, MA (1983).

[24] P.A. Macmahon, Combinatory Analysis, vols. 1 and 2, Cambridge Univ. Press, Cambridge (1915) reprinted by Chelsea, New York (1955).

[25] T. Mansour, Permutations avoiding a set of patterns from $S_{3}$ and a pattern from $S_{4}$ (preprint 1999) math.CO/9909019.

[26] T. Mansour, Permutations containing and avoiding certain patterns (preprint 1999) math.CO/9911243.

[27] T. Mansour and A. Vainshtein, Restricted permutations, continued fractions, and Chebyshev polynomials (preprint 1999) math.CO/9912052.

[28] John Noonan, The number of permutations containing exactly one increasing subsequence of length three, Discrete Mathematics, 152 (1996) 307-313.

[29] E. Pergola, G. Labelle, P. Leroux, R. Pinzani, Bell permutations and Stirling numbers interpolation, Proceedings of $11^{\text {th }}$ FPSAC, Barcelona (1999).

[30] A. Regev, Asymptotic values for degrees associated with strips of Young diagrams, Advances in Mathematics, 41 (1981) 115-136.

[31] A. Robertson, Permutations Containing and Avoiding 123 and 132 Patterns, Discrete Mathematics and Theoretical Computer Science, 3 (1999) 151-154.

[32] A. Robertson, H. Wilf and D. Zeilberger, Permutation Patterns and Continued Fractions, The Electronic Journal of Combinatorics 6 (1999).

[33] D. Rotem, Stack sortable permutations, Discrete Mathematics, 33 (1981) 185-196.

[34] R. Simion, F.W. Schmidt, Restricted permutations, European Journal of Combinatorics, 6 (1985) 383-406. 
[35] Z. Stankova, Forbidden Subsequences, Discrete Mathematics, 132 (1994) 291-316.

[36] J. West, Permutations with forbidden subsequences and stack-sortable permutations, Ph.D. Thesis, M.I.T., Cambridge, MA (1990).

[37] J. West, Sorting twice through a stack, Theoretical Computer Science, 117 (1993) 303-313.

[38] J. West, Generating trees and the Catalan and Schröder numbers, Discrete Mathematics, 146 (1995) $247-262$.

[39] J. West, Generating trees and forbidden subsequences, Discrete Mathematics, 157 (1996) 363-374. 